\title{
Oblique radiative corrections from Majorana neutrinos
}

\author{
Bernd A. Kniehl \\ II. Institut für Theoretische Physik, Universität Hamburg, Luruper Chaussee 149, 2000 Hamburg 50, Germany \\ Hans-Günter Kohrs \\ Deutsches Elektronen-Synchrotron, Notkestrasse 85, 2000 Hamburg 52, Germany \\ (Received 30 November 1992; revised manuscript received 8 March 1993)
}

\begin{abstract}
We analyze in $(S, T, U)$ space one-loop oblique (vacuum-polarization) corrections, assuming the scenario for a naturally heavy fourth-generation neutrino proposed by Hill and Paschos. Since this model suggests that the Majorana mass be of the order of the weak scale, it is crucial to employ a formulation of oblique corrections that correctly accommodates also the standard-model physics at that energy scale. We confront our results with precise data from CERN LEP and other experiments, and search the mass parameter space of the novel particles for allowed regions. We also investigate the implications for the upper bound on the top-quark mass coming from measurements of the $W$-boson mass. A heavy Majorana neutrino in the third generation decouples from electroweak physics and cannot be excluded experimentally.

PACS number(s): $13.10 .+\mathrm{q}, 12.15 . \mathrm{Ff}, 13.15 .-\mathrm{f}, 14.60 . \mathrm{Gh}$
\end{abstract}

\section{INTRODUCTION}

The experimental fact that the number of lightneutrino flavors is three [1] has ruled out the class of models that extend the standard model by naively adding new fermion generations in the traditional manner. However, reviving the idea of the seesaw mechanism, Hill and Paschos [2] suggested a scenario in which it appears quite natural that a fourth generation accommodates an unusually heavy neutral member. In fact, they could do with only two assumptions: namely, that (i) all neutrinos have Dirac masses of the same order as their charged lepton partners within a given generation and (ii) all righthanded neutrinos have Majorana masses of the order of the weak scale. In this way, they could arrange that the predicted light-neutrino masses come out acceptably small, compatible with their current upper bounds [3], and that, at the same time, the additional neutrinos are so heavy that they do not appreciably contribute to the invisible $Z$-boson width measured at the CERN $e^{+} e^{-}$collider LEP [1].

The implications at the tree level of a modified lepton sector, involving three generations with heavy Majorana neutrinos and general mixing, on a variety of electroweak observables have recently been investigated in an almost encyclopedic fashion [4]. In this case, the corrections essentially originate in the deviations of the gauge couplings from their standard-model values and can be expressed in terms of certain combinations of mixing parameters, which are experimentally constrained to be small. Loops involving the neutrinos provide $O(\alpha)$ corrections to these effects and can, as a first approximation, be neglected [4]. The logistics of direct searches for high-mass Majorana neutrinos at present and future $e p$ and $e^{+} e^{-}$colliders has been assessed in Ref. [5].

In this work, we shall focus attention on a complementary aspect of massive-neutrino phenomenology, namely, on electroweak radiative corrections induced in the HillPaschos scenario by the fourth fermion generation, which includes two Majorana neutrinos and a charged lepton. In addition, we assume that there is a conventional doublet of quarks to cancel the anomaly. To our mind, this is a very natural way of extending the minimal standard model by a novel fermion family. However, we should point out that alternative anomaly-free extensions by colorless fermions endowed with Majorana masses are conceivable [6].

In order to reduce the number of free parameters to be varied independently, we ignore the possibility of mixing between the fourth generation and the first three. Then, at the one-loop level, these novel particles can manifest themselves in electroweak physics only through contributions to the gauge-boson propagators; such corrections are customarily called oblique [7]. It is well known that oblique corrections due to new physics can be classified by means of three parameters in a general and renormalization-scheme-independent way [8-13]. The $(S, T, U)$ set by Peskin and Takeuchi [10] has been widely accepted and we shall employ it here, too. New-physicsinduced quantum corrections to the muon lifetime, embodied by the well-known quantity $\Delta r[14]$, can be expressed as a certain linear combination of $S, T$, and $U$ and the effect of a fourth fermion generation including Majorana neutrinos on $\Delta r$ has been thoroughly analyzed by Bertolini and Sirlin [15]. Our goal is to incorporate in this type of analysis the missing two dimensions. This will enable us to confront our results with the full power of accurate low-energy, LEP, and $M_{W}$ data and to establish mass bounds on the additional particles.

This paper is organized as follows. In Sec. II, we fix our notation and reexamine the definitions of $S, T$, and $U$. In Sec. III, we derive the contributions to the gaugeboson vacuum polarizations induced by a fourth lepton family with Majorana neutrinos assuming the mixing 
with the first three families to be negligible. We then evaluate the corresponding shifts in $S, T$, and $U$ and provide simple approximation formulas appropriate for the limit of ultraheavy leptons. We also briefly comment on the oblique corrections due to a heavy Majorana neutrino in the third fermion generation. The vacuum polarizations due to a conventional heavy-quark doublet are well known (see, e.g., Ref. [16]) and the corresponding contributions to $S, T$, and $U$ emerge without more ado from the master formulas presented in Sec. II, so that we need not list them explicitly. In Sec. IV, we scan the multidimensional space of the novel fermion masses for experimentally allowed ranges by requiring that $S, T$, and $U$ simultaneously satisfy the constraints imposed by a recent global analysis of LEP and other data [17]. We also study how the range of $m_{t}$ values favored by the measurement of $M_{W}$ depends on the additional mass parameters. Our conclusions are summarized in Sec. V.

\section{FORMALISM}

In the following, we shall work in the electroweak onmass-shell (OMS) scheme [14,18]. In this scheme it is particularly evident that oblique radiative corrections can be parametrized in terms of four independent quantities which have the property that they are automatically ul- traviolet finite. Such quantities are sometimes called $o b$ servable radiative corrections [19]. Since one of them resides in the purely electromagnetic sector of the standard electroweak theory, there remain three variables to characterize the more exciting genuinely weak phenomena and, in particular, to probe new physics beyond the standard model. Such three-dimensional parametrizations have gained vogue some time ago and meanwhile there exits a confusing wealth of variant definitions and notations [8-13], which have been nicely catalogued in Ref. [20]. Perhaps, the most popular language is the one by Peskin and Takeuchi [10], who coined the $(S, T, U)$ terminology. However, these authors were mainly concerned with new physics at energy far above the weak scale and their original definitions of $S$ and $U$ lead to mass singularities when they are naively applied to the standard model itself. The same is true of the $\left(\varepsilon_{1}, \varepsilon_{2}, \varepsilon_{3}\right)$ set introduced by Altarelli and Barbieri [13]. To overcome this problem, one eliminates the derivatives, evaluated at zero-momentum transfer, of the non-QED vacuum-polarization functions by suitably reintroducing the weak-boson masses [11,12,21].

As mentioned above, observable radiative corrections can be identified quite systematically in the OMS scheme. Toward this end, one considers the transverse gaugeboson vacuum-polarization functions in their renormalized forms:

$$
\begin{aligned}
& \hat{\Pi}_{W W}\left(q^{2}\right)=\Pi_{W W}\left(q^{2}\right)-\operatorname{Re} \Pi_{W W}(w)+\left(q^{2}-w\right)\left[\left(c^{2} / s^{2}\right) R-\Pi_{A A}^{\prime}(0)\right], \\
& \hat{\Pi}_{Z Z}\left(q^{2}\right)=\Pi_{Z Z}\left(q^{2}\right)-\operatorname{Re} \Pi_{Z Z}(z)+\left(q^{2}-z\right)\left[\left(\frac{c^{2}}{s^{2}}-1\right) R-\Pi_{A A}^{\prime}(0)\right], \\
& \hat{\Pi}_{Z A}\left(q^{2}\right)=\Pi_{Z A}\left(q^{2}\right)-\Pi_{Z A}(0)-q^{2}(c / s) R \\
& \hat{\Pi}_{A A}\left(q^{2}\right)=\Pi_{A A}\left(q^{2}\right)-q^{2} \Pi_{A A}^{\prime}(0)
\end{aligned}
$$

where $q$ denotes the external four-momentum and

$$
R=\operatorname{Re}\left(-\frac{\Pi_{W W}(w)}{w}+\frac{\Pi_{Z Z}(z)}{z}\right)-2 \frac{s}{c} \frac{\Pi_{Z A}(0)}{z} .
$$

Here and in the following, we use the shorthand notation $w=M_{W}^{2}, z=M_{Z}^{2}$, and $c^{2}=1-s^{2}=w / z$. The standardmodel expressions in $O(\alpha)$ for the unrenormalized $\Pi$ functions may be found, for instance, in Ref. [16]; perturbative $\boldsymbol{O}\left(\alpha \alpha_{s}\right)$ corrections to these functions are listed in Ref. [22]. As per construction, Eq. (2.1) satisfies the onshell conditions

$\operatorname{Re}_{W W}(w)=\operatorname{Re} \hat{\Pi}_{Z Z}(z)=\hat{\Pi}_{Z A}(0)=\widehat{\Pi}_{A A}(0)=0$.

On the other hand, $\hat{\Pi}_{W W}(0), \hat{\Pi}_{Z Z}(0), \operatorname{Re}_{Z A}(z)$, and $\operatorname{Re}_{A A}(z)$ represent nontrivial and manifestly finite combinations of unrenormalized two-point functions, which are clearly renormalization-scheme independent. They bear the following physical meanings: (i) $\Delta r_{\text {oblique }}=\bigcap_{W W}(0) / w$ embodies the propagator corrections to the muon lifetime [14];
$\Delta \rho=\widehat{\Pi}_{Z Z}(0) / z-\widehat{\Pi}_{W W}(0) / w$ measures the quantum corrections to the ratio, $\rho=1 /(1-\Delta \rho)$, of the neutraland charged-current amplitudes at low energy [23]; (iii) $\Delta \kappa=-(c / s) \operatorname{Re} \widehat{\Pi}_{Z A}(z) / z$ controls the effective weak mixing angle, $\bar{s}^{2}=s^{2}(1+\Delta \kappa)$, that occurs in the vector coupling of the $Z \rightarrow f \bar{f}$ decay rate [18]; (iv) $\Delta \alpha=-\operatorname{Re}_{A A}(z) / z$ determines the running fine-structure constant at the $Z$-boson scale, $\bar{\alpha}=\alpha /(1-\Delta \alpha)$, where $\alpha$ is the corresponding value at the electron scale [3]. The term $\Delta \alpha$ receives significant contributions only from charged particles with masses below the $Z$-boson scale, while high-mass particles are known to decouple from it [24]. Thus, $\Delta \alpha$ is well under control and we shall not be concerned with it in the remainder of this work. Furthermore, we subtract from $\Delta r_{\text {oblique }}$ its pure QED portion by setting

$$
\left(1-\Delta r_{\text {oblique }}\right)=(1-\Delta \alpha)\left(1-\Delta r_{W}\right),
$$

so that $\Delta r_{W}$ is devoid of mass singularities when charged-particle masses are sent to zero.

The conventional parameters $S, T$, and $U$, or, 
equivalently, $\varepsilon_{1}, \varepsilon_{2}$, and $\varepsilon_{3}$, may be recovered as linear combinations of $\Delta r_{W}, \Delta \rho$, and $\Delta \kappa$ : namely,

$$
\begin{aligned}
& \alpha S=4 s^{2} \varepsilon_{3}=4 s^{2}\left[c^{2}\left(\Delta r_{W}+\Delta \rho\right)+\left(c^{2}-s^{2}\right) \Delta \kappa\right], \\
& \alpha T=\varepsilon_{1}=\Delta \rho \\
& \alpha U=-4 s^{2} \varepsilon_{2}=4 s^{2}\left[s^{2}\left(\Delta r_{W}+2 \Delta \kappa\right)-c^{2} \Delta \rho\right] .
\end{aligned}
$$

Conversely,

$$
\begin{aligned}
\Delta r_{W} & =\frac{\alpha}{s^{2}}\left[\frac{S}{2}-c^{2} T-\frac{1}{4}\left[\frac{c^{2}}{s^{2}}-1\right] U\right] \\
& =-\frac{c^{2}}{s^{2}} \varepsilon_{1}+\left(\frac{c^{2}}{s^{2}}-1\right] \varepsilon_{2}+2 \varepsilon_{3}, \\
\Delta \kappa & =\frac{\alpha}{s^{2}}\left[-\frac{S}{4}+c^{2} T+\frac{c^{2}}{4 s^{2}} U\right] \\
& =\frac{c^{2}}{s^{2}}\left(\varepsilon_{1}-\varepsilon_{2}\right)-\varepsilon_{3} .
\end{aligned}
$$

It is well known that $T$ quantifies the violation of the custodial isospin symmetry, while $S$ measures the size of the Higgs sector and counts the total number of weak doublets. In many new-physics scenarios, $U$ plays a fairly unimportant role and it is explicitly fixed to zero by some authors. However, models with anomalous $W$-boson interactions provide a counterexample to this rule [13].

\section{ONE-LOOP RESULTS}

In this section, we analyze how the fourth-generation leptons of the Hill-Paschos scenario would manifest themselves in $(S, T, U)$ space. For simplicity, we neglect mixing between the fourth generation and the first three. The extension to the general case is straightforward [25]. We shall allow for a heavy Majorana neutrino in the third generation at the end of this section. The shifts in $S, T$, and $U$ induced by the fourth-generation quark doublet may be obtained by inserting their well-known vacuum polarizations [16] into Eq. (2.4) in conjunction with Eqs. (2.1), (2.2), and items (i) -(iv) and need not be listed here. When the Hill-Paschos scenario is to be confronted with the data the quarkonic contribution must be included in order for the phenomenological analysis to be selfconsistent and as realistic as possible.

Bertolini and Sirlin [15] have formulated a nice representation for the leptonic sector of the Hill-Paschos scenario and we shall largely adopt their conventions. Their model contains a doublet of Majorana fields,

$$
N=\left(\begin{array}{l}
N_{1} \\
N_{2}
\end{array}\right)
$$

with a mass term

$$
\mathcal{L}_{M}=-\frac{1}{2} \bar{N}\left(\begin{array}{cc}
m_{1} & 0 \\
0 & m_{2}
\end{array}\right) N .
$$

The two mass eigenstates $N_{1}$ and $N_{2}$ are related to the left- and right-handed neutrinos $v_{L}$ and $v_{R}$ by

$$
\left(v_{L} v_{R}^{c}\right)=\left(\begin{array}{cc}
i c_{\theta} & s_{\theta} \\
-i s_{\theta} & c_{\theta}
\end{array}\right) \frac{1-\gamma_{5}}{2}\left(\begin{array}{l}
N_{1} \\
N_{2}
\end{array}\right) \text {, }
$$

where $v_{R}^{c}=C\left(\bar{v}_{R}\right)^{T}$ is the (left-handed) charge conjugate of $v_{R}$ and $c_{\theta}^{2}=1-s_{\theta}^{2}=m_{2} /\left(m_{1}+m_{2}\right)$. Equation (3.2) corresponds to a Dirac mass, $m_{D}=\sqrt{m_{1} m_{2}}$, for both $v_{L}$ and $v_{R}$ and, in addition, to a Majorana mass, $M=m_{2}-m_{1}$, for $v_{R}$. The lepton family is completed by a negatively charged lepton field $l$ with mass $m_{l}$. It is understood that $v_{L}$ and $l_{L}$ are the up- and down-type components of a doublet of weak isospin, respectively, while $v_{R}$ and $l_{R}$ each form a singlet. The charged- and neutral-current interactions of the leptons are described by the Lagrangian density

$$
\begin{aligned}
\mathcal{L}_{I}= & \frac{e}{\sqrt{2} s} W_{\mu} \bar{l}_{L} \gamma^{\mu} v_{L}+\text { H.c. } \\
& +\frac{e}{c s} Z_{\mu}\left(\frac{1}{2} \bar{v}_{L} \gamma^{\mu} v_{L}-\frac{1}{2} \bar{l}_{L} \gamma^{\mu} l_{L}+s^{2} \bar{l} \gamma^{\mu} l\right],
\end{aligned}
$$

where $e=\sqrt{4 \pi \alpha}$ is the positron charge. The case of one Dirac neutrino, with mass $m_{D}$, is recovered for $M=0$.

We are now ready to derive the contributions to the $W$ and $Z$-boson self-energies induced by $N_{1}, N_{2}$, and $l$ at one loop. Using dimensional regularization, we find

$$
\begin{aligned}
& \Pi_{W W}\left(q^{2}\right)=\frac{e^{2}}{8 s^{2}}\left[c_{\theta}^{2}\left(\Pi^{V}+\Pi^{A}\right)\left(q^{2}, m_{1}, m_{l}\right)\right. \\
& \left.+s_{\theta}^{2}\left(\Pi^{V}+\Pi^{A}\right)\left(q^{2}, m_{2}, m_{l}\right)\right], \\
& \begin{aligned}
\Pi_{Z Z}\left(q^{2}\right)=\frac{e^{2}}{16 c^{2} s^{2}} & {\left[2 c_{\theta}^{4} \Pi^{A}\left(q^{2}, m_{1}, m_{1}\right)\right.} \\
& +2 s_{\theta}^{4} \Pi^{A}\left(q^{2}, m_{2}, m_{2}\right) \\
& +4 c_{\theta}^{2} s_{\theta}^{2} \Pi^{V}\left(q^{2}, m_{1}, m_{2}\right) \\
& \left.+v_{l}^{2} \Pi^{V}\left(q^{2}, m_{l}, m_{l}\right)+\Pi^{A}\left(q^{2}, m_{l}, m_{l}\right)\right],
\end{aligned}
\end{aligned}
$$

where $v_{l}=4 s^{2}-1$ and

$$
\begin{aligned}
\Pi^{V, A}\left(q^{2}, m_{1}, m_{2}\right)=\frac{1}{12 \pi^{2}}[ & {\left[s-\frac{m_{1}^{2}+m_{2}^{2}}{2} \pm 3 m_{1} m_{2}-\frac{\left(m_{1}^{2}-m_{2}^{2}\right)^{2}}{2 q^{2}}\right] B_{0}\left(q^{2}, m_{1}^{2}, m_{2}^{2}\right) } \\
& +m_{1}^{2}\left[-1+\frac{m_{1}^{2}-m_{2}^{2}}{2 q^{2}}\right] B_{0}\left(0, m_{1}^{2}, m_{1}^{2}\right)+m_{2}^{2}\left[-1+\frac{m_{2}^{2}-m_{1}^{2}}{2 q^{2}}\right] B_{0}\left(0, m_{2}^{2}, m_{2}^{2}\right) \\
& \left.-\frac{q^{2}}{3}+\frac{\left(m_{1}^{2}-m_{2}^{2}\right)^{2}}{2 q^{2}}\right]
\end{aligned}
$$


An expression for the standard scalar one-loop integral, $\boldsymbol{B}_{0}\left(q^{2}, m_{1}^{2}, m_{2}^{2}\right)$, may be found, for example, in Ref. [16]. Note that this function contains an ultraviolet-divergent term of the form

$$
\Delta=2 /(4-n)-\ln \left(m_{1} m_{2} / \mu^{2}\right),
$$

where $n$ is the dimensionality of space-time and $\mu$ is the 't Hooft mass. For completeness, we also list the contributions to the residual vacuum polarizations due to $l$ :

$$
\begin{aligned}
& \Pi_{Z A}\left(q^{2}\right)=\frac{e^{2} v_{l}}{4 c s} \Pi^{V}\left(q^{2}, m_{l}, m_{l}\right), \\
& \Pi_{A A}\left(q^{2}\right)=e^{2} \Pi^{V}\left(q^{2}, m_{l}, m_{l}\right) .
\end{aligned}
$$

The poles in $(n-4)$ and the $\mu$ dependence of Eqs. (3.5) and (3.7) cancel when observable radiative corrections are calculated. We note in passing that the integral formula for $\Delta r_{\text {oblique }}$ given by Eq. (29) of Ref. [15] agrees with the analytic representation on the basis of our Eqs. (2.1), (3.5), and (3.7).

Using the definitions collected in the previous section, we obtain

$$
\begin{aligned}
T=\frac{1}{16 \pi s^{2} w}\{ & m_{l}^{2}+\frac{m_{1} m_{2}}{\left(m_{1}+m_{2}\right)^{2}}\left[6 m_{1} m_{2}+\frac{m_{1}^{4}+m_{2}^{4}-2 m_{1} m_{2}\left(m_{1}^{2}+m_{2}^{2}\right)}{m_{1}^{2}-m_{2}^{2}} \ln \frac{m_{1}^{2}}{m_{2}^{2}}\right] \\
& \left.+\frac{m_{1} m_{2}}{m_{1}+m_{2}}\left[\left\{m_{2}-m_{1} \frac{m_{1}^{2}+m_{l}^{2}}{m_{1}^{2}-m_{l}^{2}}\right] \ln \frac{m_{1}^{2}}{m_{l}^{2}}+\left[m_{1}-m_{2} \frac{m_{2}^{2}+m_{l}^{2}}{m_{2}^{2}-m_{l}^{2}}\right] \ln \frac{m_{2}^{2}}{m_{l}^{2}}\right]\right\}
\end{aligned}
$$

and further

$$
\begin{gathered}
S=\frac{\pi}{z}\left\{-2 c_{\theta}^{4}\left[\operatorname{Re}^{A}\left(z, m_{1}, m_{1}\right)-\Pi^{A}\left(0, m_{1}, m_{1}\right)\right]-2 s_{\theta}^{4}\left[\operatorname{Re}^{A}\left(z, m_{2}, m_{2}\right)-\Pi^{A}\left(0, m_{2}, m_{2}\right)\right]\right. \\
\left.-4 c_{\theta}^{2} s_{\theta}^{2}\left[\operatorname{Re} \Pi^{V}\left(z, m_{1}, m_{2}\right)-\Pi^{V}\left(0, m_{1}, m_{2}\right)\right]+3 \operatorname{Re}^{V}\left(z, m_{l}, m_{l}\right)-\operatorname{Re}^{A}\left(z, m_{l}, m_{l}\right)+\Pi^{A}\left(0, m_{l}, m_{l}\right)\right\}, \\
S+U=2 \pi\left\{-\frac{c_{\theta}^{2}}{w}\left[\operatorname{Re}\left(\Pi^{V}+\Pi^{A}\right)\left(w, m_{1}, m_{l}\right)-\left(\Pi^{V}+\Pi^{A}\right)\left(0, m_{1}, m_{l}\right)\right]\right. \\
\left.-\frac{s_{\theta}^{2}}{w}\left[\operatorname{Re}\left(\Pi^{V}+\Pi^{A}\right)\left(w, m_{2}, m_{l}\right)-\left(\Pi^{V}+\Pi^{A}\right)\left(0, m_{2}, m_{l}\right)\right]+\frac{2}{z} \operatorname{Re}^{V}\left(z, m_{l,} m_{l}\right)\right\},
\end{gathered}
$$

from which $U$ follows. We stress that Eqs. (3.8) and (3.9) are valid for arbitrary values of $m_{1}, m_{2}$, and $m_{l}$. In particular, they do not develop singularities when one or more fermion masses are sent to zero. For $m_{1}, m_{2}, m_{l} \gg M_{Z}, S$ and $U$ can be approximated by

$$
\begin{aligned}
& S=\frac{1}{6 \pi} \frac{1}{m_{1}+m_{2}}[\left.\frac{1}{m_{1}+m_{2}}\left[\frac{3}{2}\left(m_{1}^{2}+m_{2}^{2}\right)+\frac{m_{1} m_{2}}{3}+A\right]+m_{2} \ln \frac{m_{1}^{2}}{m_{l}^{2}}+m_{1} \ln \frac{m_{2}^{2}}{m_{l}^{2}}\right], \\
& U=\frac{1}{6 \pi} \frac{1}{m_{1}+m_{2}}\left[-\frac{1}{m_{1}+m_{2}}\left[\frac{13}{6}\left(m_{1}^{2}+m_{2}^{2}\right)+\frac{5}{3} m_{1} m_{2}+A\right]\right. \\
&+\frac{m_{2}}{\left(m_{1}^{2}-m_{l}^{2}\right)^{2}}\left[4 m_{1}^{2} m_{l}^{2}+\frac{\left(m_{1}^{2}+m_{l}^{2}\right)\left(m_{1}^{4}+m_{l}^{4}-4 m_{1}^{2} m_{l}^{2}\right)}{m_{1}^{2}-m_{l}^{2}} \ln \frac{m_{1}^{2}}{m_{l}^{2}}\right] \\
&\left.+\frac{m_{1}}{\left(m_{2}^{2}-m_{l}^{2}\right)^{2}}\left[4 m_{2}^{2} m_{l}^{2}+\frac{\left(m_{2}^{2}+m_{l}^{2}\right)\left(m_{2}^{4}+m_{l}^{4}-4 m_{2}^{2} m_{l}^{2}\right)}{m_{2}^{2}-m_{l}^{2}} \ln \frac{m_{2}^{2}}{m_{l}^{2}}\right]\right],
\end{aligned}
$$

where

$$
A=\frac{m_{1}^{2} m_{2}^{2}}{\left(m_{1}^{2}-m_{2}^{2}\right)^{2}}\left[-3\left(m_{1}^{2}+m_{2}^{2}\right)+4 m_{1} m_{2}\right]+\frac{m_{1} m_{2}}{m_{1}^{2}-m_{2}^{2}}\left[m_{1}^{2}+m_{2}^{2}+\frac{2 m_{1}^{2} m_{2}^{2}}{\left(m_{1}^{2}-m_{2}^{2}\right)^{2}}\left(-m_{1}^{2}-m_{2}^{2}+3 m_{1} m_{2}\right)\right] \ln \frac{m_{1}^{2}}{m_{2}^{2}} .
$$

The ultraheavy-fermion limit has been considered also in Ref. [26]. We shall see in the next section that there is an extensive range of plausible mass values where the approximation by Eqs. (3.10) and (3.11) varies from crude to completely inadequate and the exact formula (3.9), which, to our knowledge, cannot be found elsewhere in the literature, is requisite in order to acquire reliable predictions. 
We close this section by taking a brief look at oblique radiative corrections due to the third lepton family allowing for the extension by a high-mass Majorana neutrino, with $m_{2} \gtrsim M_{W}$. In this case, $l$ is identified with the familiar $\tau$ lepton, $N_{1}$ replaces the associated Dirac neutrino, $v_{\tau}$, and $N_{2}$ represents the novel Majorana neutrino. As explained above, we may safely neglect $m_{l}$ and $m_{1}$ relative to the weak scale (and $m_{2}$ ) without encountering any mass singularities. To be specific, the affected terms are suppressed by powers of $m_{l}^{2} / w, m_{l} / m_{2}$, and similarly for $m_{1}$. We emphasize that this limit cannot be taken in the approximation formula (3.10), which again demonstrates the quest for a general formulation of observable radiative corrections such as the one proposed in Sec. II. Detailed inspection of Eqs. (3.8) and (3.9) reveals that the considered modification of the third lepton family cannot be distinguished from the original form in the minimal standard model, the results in both cases being

$$
\begin{aligned}
& S=T=0, \\
& U=(1 / 3 \pi) \ln c^{2},
\end{aligned}
$$

up to very small terms. This means that $N_{2}$ decouples from electroweak physics at one loop, so that present achievements in high-precision experimentation are not yet powerful enough to put a handle on it.

\section{PHENOMENOLOGY}

In this section we explore the phenomenological consequences of our results. We assume that the standard model is extended by a fourth generation which consists of leptons, $N_{1}, N_{2}$, and $l$, as described at the outset of Sec. III and quarks, $T$ and $B$, with similar interactions and quantum numbers as the up- and down-type quarks, respectively, in the first three generations. Inspired by the fact that the third-generation quarks participate only feebly in the Cabibbo-Kobayashi-Maskawa mixing, we ignore the possibility of mixing between the established and the novel flavors. To avoid pair production of the new particles from $Z$-boson decay, we assume $m_{1}, m_{2}, m_{l}, m_{T,} m_{B}>M_{Z} / 2$. This also impedes their associated production from $W$-boson decay. Furthermore, it appears natural to impose the hierarchy $m_{T}>m_{t}$.

To demonstrate the importance of a formulation of observable radiative corrections that properly accommodates the physics at the weak scale, we compare in Fig. 1 the full contributions to $S$ and $U$ induced by $N_{1}, N_{2}$, and $l$ [cf. Eq. (3.9)] with their ultraheavy-lepton limits [cf. Eq. (3.10)], assuming $m_{1}=\xi \times 50 \mathrm{GeV}, m_{2}=\xi \times 150 \mathrm{GeV}$, and $m_{l}=\xi \times 100 \mathrm{GeV}$, with $1 \leq \xi \leq 5$. While the asymptotic expansions are manifestly scale invariant, the exact results exhibit a dramatic $\xi$ dependence at $\xi \gtrsim 1$. In particular, the sign of $U$ changes and its magnitude increases by more than $300 \%$ as compared to its asymptotic value. In the following, we shall always employ our rigorous results of Eq. (3.9).

Next, we explore the dependence of $S, T$, and $U$ on the three independent mass parameters $m_{D}=\sqrt{m_{1} m_{2}}$, $M=m_{2}-m_{1}$, and $m_{l}$. In Fig. 2, we set $M=0$, which

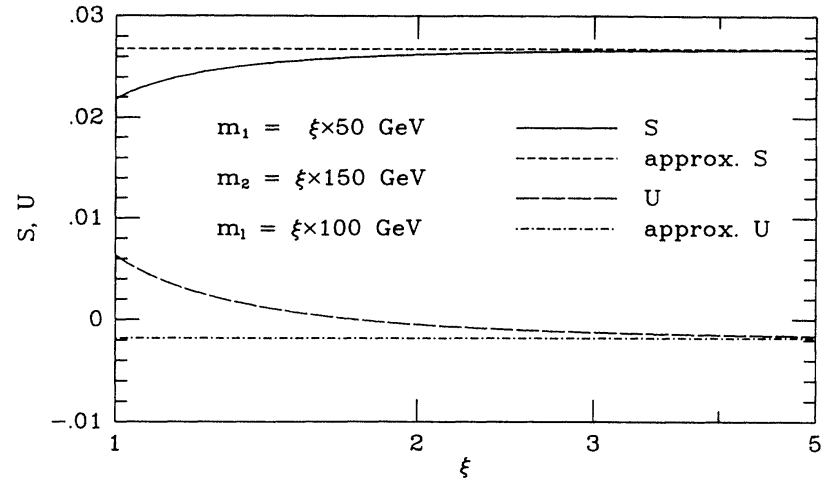

FIG. 1. Contributions to $S$ and $U$ due to fourth-generation leptons, $N_{1}, N_{2}$, and $l$, with masses $m_{1}=\xi \times 50 \mathrm{GeV}$, $m_{2}=\xi \times 150 \mathrm{GeV}$, and $m_{l}=\xi \times 100 \mathrm{GeV}$, as functions of $\xi$. The exact results of Eq. (3.9) are compared with their asymptotic expansions of Eq. (3.10).

corresponds to the case of one Dirac neutrino with mass $m_{D}$, and plot $S, T$, and $U$ over the $\left(m_{D}, m_{l}\right)$ plane. We observe that $S$ can take both positive and negative values and becomes approximately antisymmetrical in $m_{D}$ and $m_{l}$ for $m_{D}, m_{l} \gg M_{Z} / 2$. Contrarily, $T$ and $U$ are exactly symmetrical in $m_{D}$ and $m_{l}$. Of course, $T=0$ for $m_{D}=m_{l}$ and $T>0$ otherwise. Similarly, $U$ is positive in the considered $\left(m_{D}, m_{l}\right)$ range and close to zero for $m_{D}=m_{l}$. In Fig. 3, we choose $m_{l}=100 \mathrm{GeV}$ and display $S, T$, and $U$ over the $\left(m_{D}, M\right)$ plane. The contours with $M=0$ in this figure correspond to the contours with $m_{l}=100 \mathrm{GeV}$ in Fig. 2. Note that

$$
m_{D}<\left(M_{Z} / 2\right) \sqrt{1+2 M / M_{Z}}
$$

implies $m_{1}<M_{Z} / 2$ and thus defines the range excluded by the measurements of the invisible $Z$-boson width [1]. In particular, the striking threshold features in Figs. 3(a) and $3(\mathrm{c})$ are located within this range. Sufficiently far away from this range $S, T$, and $U$ all increase (decrease) with increasing $m_{D}(M)$. It is interesting to observe that for nonvanishing $M$ both $T$ and $U$ can take negative values in the mass range of interest. We shall see later that in the case of the $T$ parameter this property can be exploited to increase the $m_{t}$ upper bound or to compensate for the effect of isospin breaking in the $(T, B)$ doublet.

We are now ready to determine the allowed mass range for the novel particles by confronting our results with the collective power of the latest low-energy, LEP, and $M_{W}$ data. Assuming the reference values $m_{t}=130 \mathrm{GeV}$ and $M_{H}=M_{Z}$, a very recent global analysis [17] has yielded the following contributions to $\varepsilon_{1}, \varepsilon_{2}$, and $\varepsilon_{3}$ from beyond the standard model:

$$
\begin{aligned}
& \delta \varepsilon_{1}=-(0.09 \pm 0.25) \times 10^{-2}, \\
& \delta \varepsilon_{2}=(0.09 \pm 0.72) \times 10^{-2}, \\
& \delta \varepsilon_{3}=-(0.24 \pm 0.38) \times 10^{-2} .
\end{aligned}
$$


By demanding that these contributions are simultaneously generated by $N_{1}, N_{2}, l, T$, and $B$, we can determine the range of $m_{D}, \boldsymbol{M}, m_{l}, m_{T}$, and $m_{B}$ currently favored by experiment. Since we are mainly interested in the lepton-
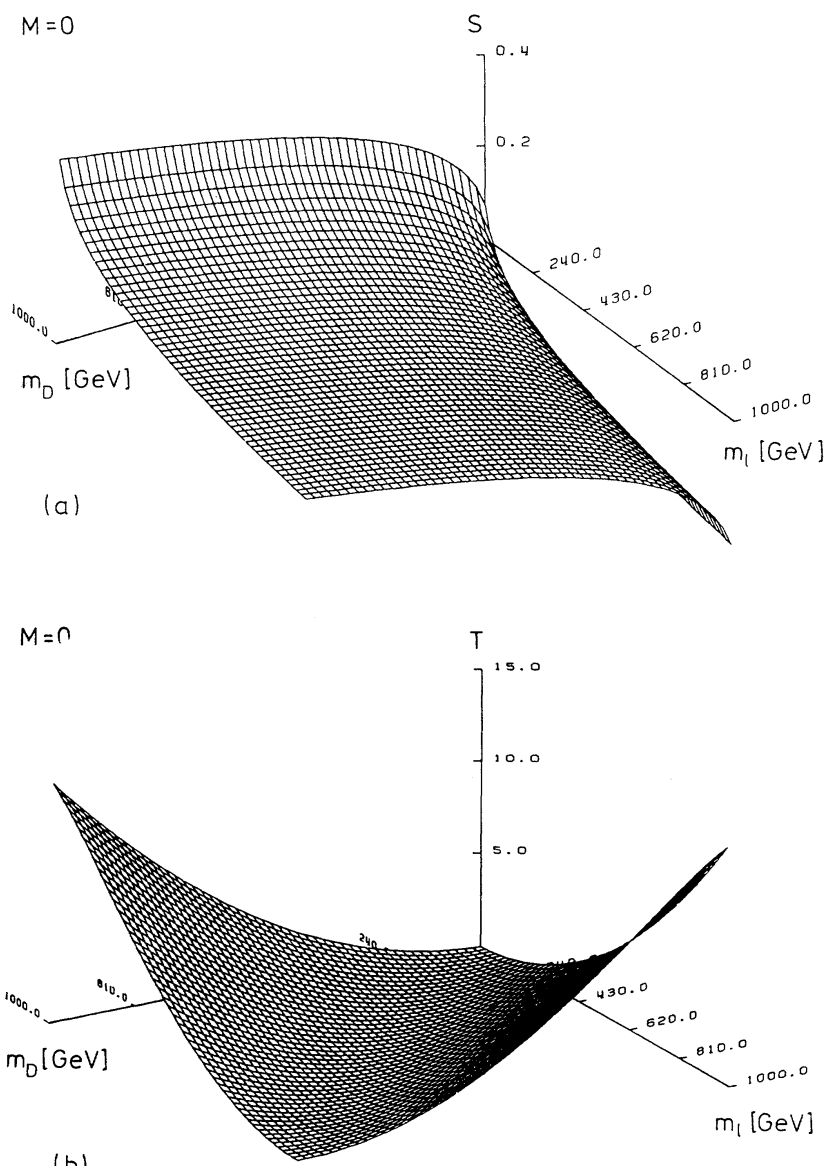

(b)



FIG. 2. Contributions to (a) $S$, (b) $T$, and (c) $U$ due to fourthgeneration leptons, $N_{1}, N_{2}$, and $l$, as functions of $m_{D}$ and $m_{l}$, assuming $m_{1}=m_{2}=m_{D}$ (Dirac neutrino). ic sector, we select $m_{T}=m_{B}=150 \mathrm{GeV}$ for the time being to reduce this multidimensional problem. Then, we can fix $M$ and search for points in the $\left(m_{D}, m_{l}\right)$ plane that comply with Eq. (4.1). This is done for $M=0,100,300$,
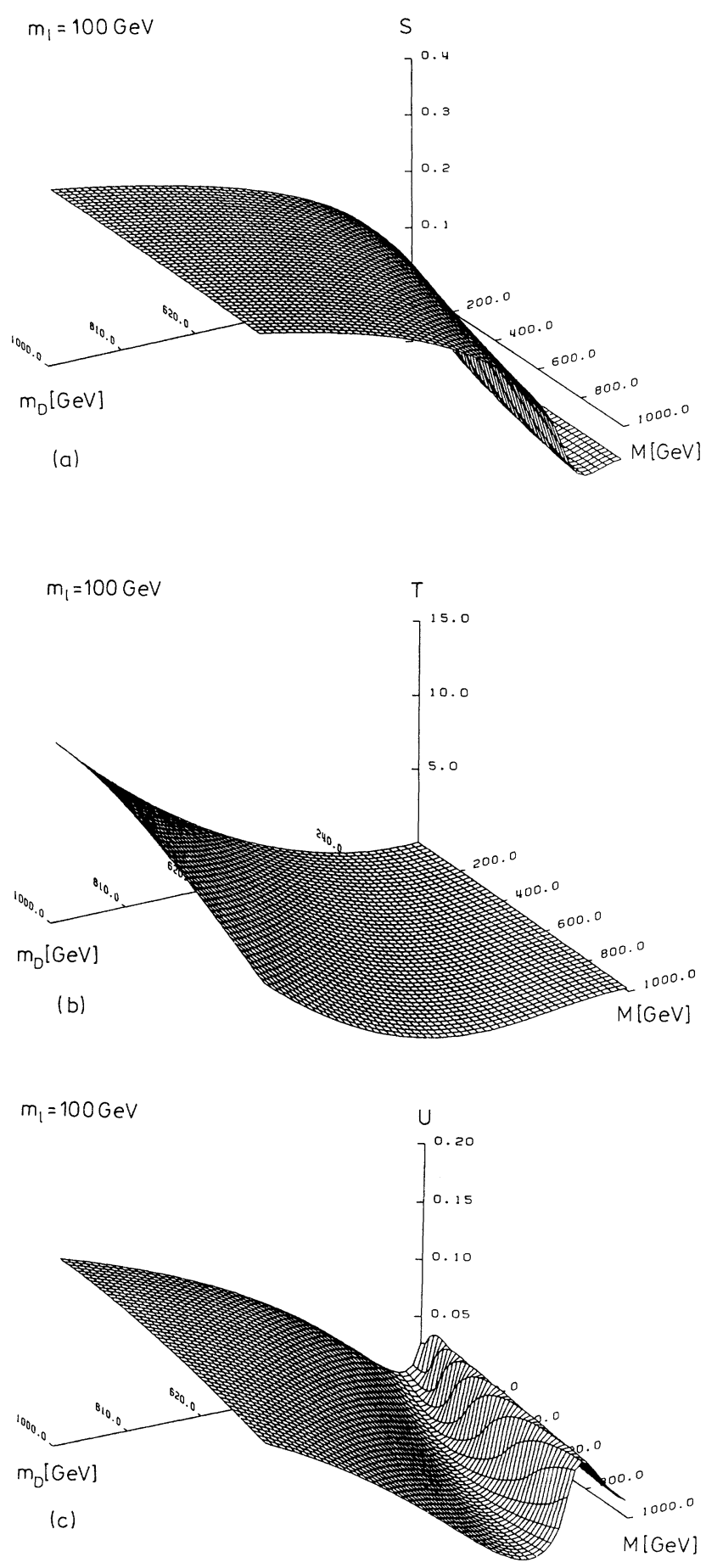

FIG. 3. Contributions to (a) $S$, (b) $T$, and (c) $U$ due to fourthgeneration leptons, $N_{1}, N_{2}$, and $l$, with $m_{l=100 \mathrm{GeV}}$, as functions of $m_{D}=\sqrt{m_{1} m_{2}}$ and $M=m_{2}-m_{1}$ (Majorana neutrinos). 

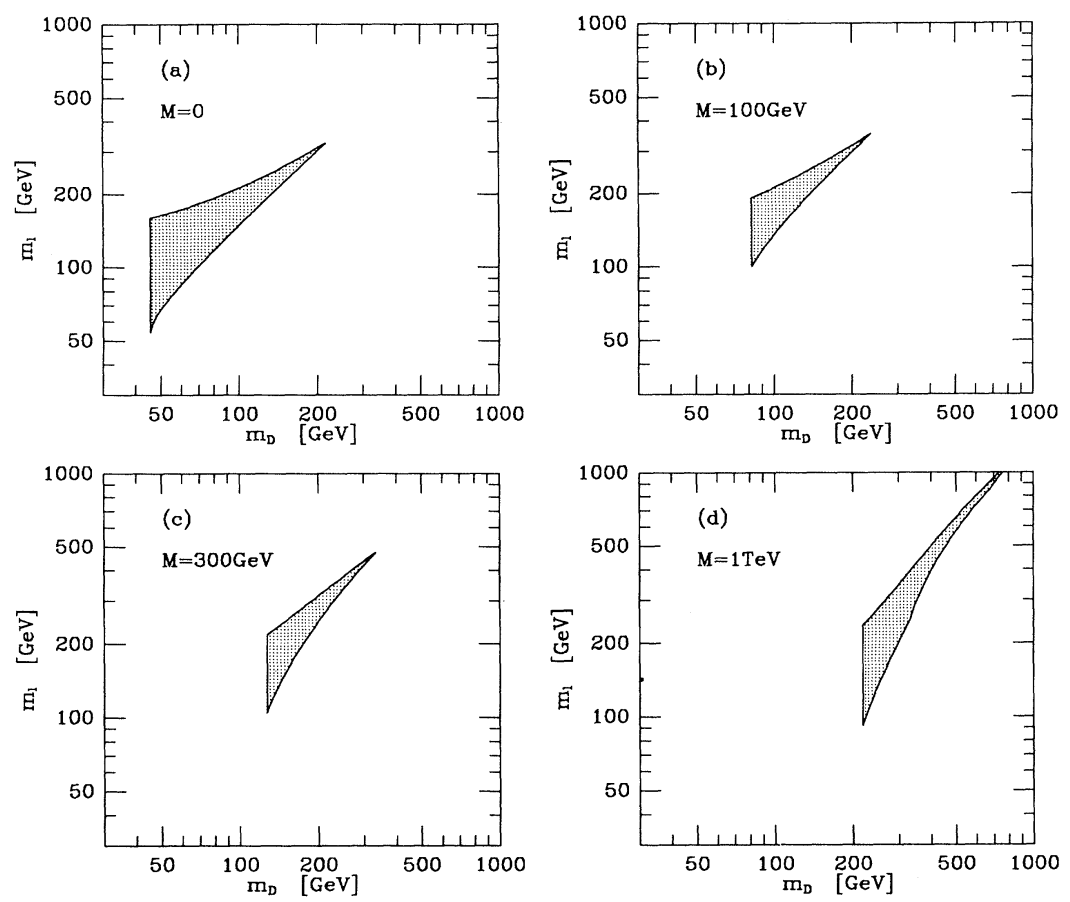

FIG. 4. Range of $m_{D}$ and $m_{l}$ experimentally allowed at the $68 \%$ confidence level, assuming $m_{t}=130 \mathrm{GeV}, M_{H}=M_{Z}, m_{T}=m_{B}=150$ $\mathrm{GeV}$, and (a) $M=0$, (b) $M=100 \mathrm{GeV}$, (c) $M=300 \mathrm{GeV}$, and (d) $M=1 \mathrm{TeV}$. and $1000 \mathrm{GeV}$ in Fig. 4, where the hatched areas are compatible with experiment at the $68 \%$ confidence level. As might be expected, $\varepsilon_{2}$, or, equivalently, $U$ is irrelevant for this type of analysis, since it is theoretically predicted to be smaller and experimentally much less constrained than the other two parameters. For each value of $M$ the lower bound on $m_{D}$ follows from the requirement that $m_{1}>M_{Z} / 2$. For increasing $M$ the allowed zone turns into a narrow stripe, extending out to larger values of $m_{D}$ and $m_{l}$, and can possibly be ruled out by appealing to fine-tuning problems. Moreover, one should bear in mind that $m_{D}$ and $m_{l}$ derive their origins from the Higgs mechanism and are thus constrained from above by renormalization-group triviality bounds [27]. We conclude that values of $M$ considerably in excess of $1 \mathrm{TeV}$ are disfavored by experiment.

Next, we allow for isospin breaking in the quark doublet by setting $m_{T}=200 \mathrm{GeV}$ and $m_{B}=100 \mathrm{GeV}$ and re- peat the analysis of Fig. 4; see Fig. 5. In the Dirac neutrino case $(M=0)$ such a $(T, B)$ doublet is excluded experimentally by its oversized positive contribution to the $T$ parameter [23],

$$
T=\frac{N_{c}}{16 \pi s^{2} w}\left[m_{T}^{2}+m_{B}^{2}-\frac{2 m_{T}^{2} m_{B}^{2}}{m_{T}^{2}-m_{B}^{2}} \ln \frac{m_{T}^{2}}{m_{B}^{2}}\right],
$$

which can be read off from Fig. 2(b) after the replacements $m_{D} \rightarrow m_{T}$ and $m_{l} \rightarrow m_{B}$ and multiplication by the color factor, $N_{c}=3$. However, from Fig. 3(b) it is evident that for sufficiently large $M$ there exists a range of $m_{D}$ and $m_{l}$ values for which the lepton sector is able to reduce the $T$ value below its experimental upper bound. In the case under consideration this happens at $M \approx 270$ $\mathrm{GeV}$ and thus we do not show the cases $M=0$ and 100 $\mathrm{GeV}$ in Fig. 5. On the other hand, from the ultraheavyquark approximation formula [10]
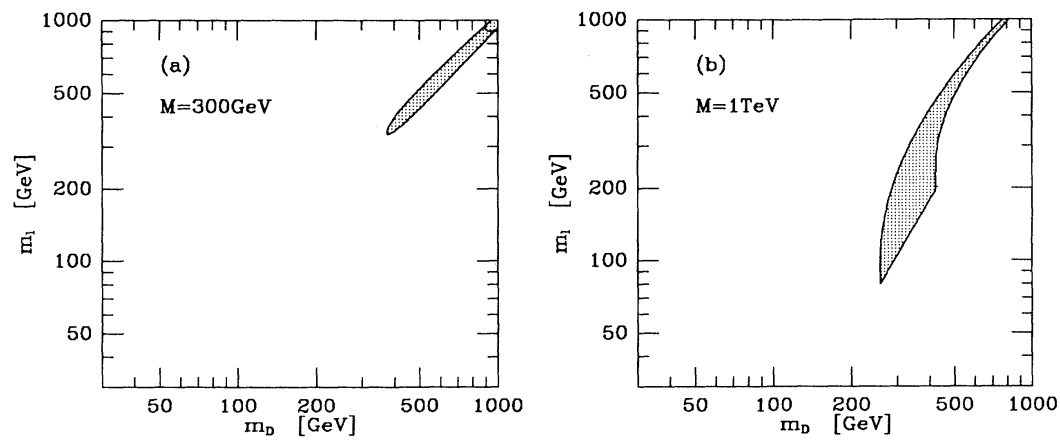

FIG. 5. Range of $m_{D}$ and $m_{l}$ experimentally allowed at the $68 \%$ confidence level, assuming $m_{t}=130 \mathrm{GeV}, M_{H}=M_{Z}, m_{T}=200 \mathrm{GeV}$, $m_{B}=100 \mathrm{GeV}$, and (a) $\boldsymbol{M}=300 \mathrm{GeV}$, and (b) $M=1 \mathrm{TeV}$ 

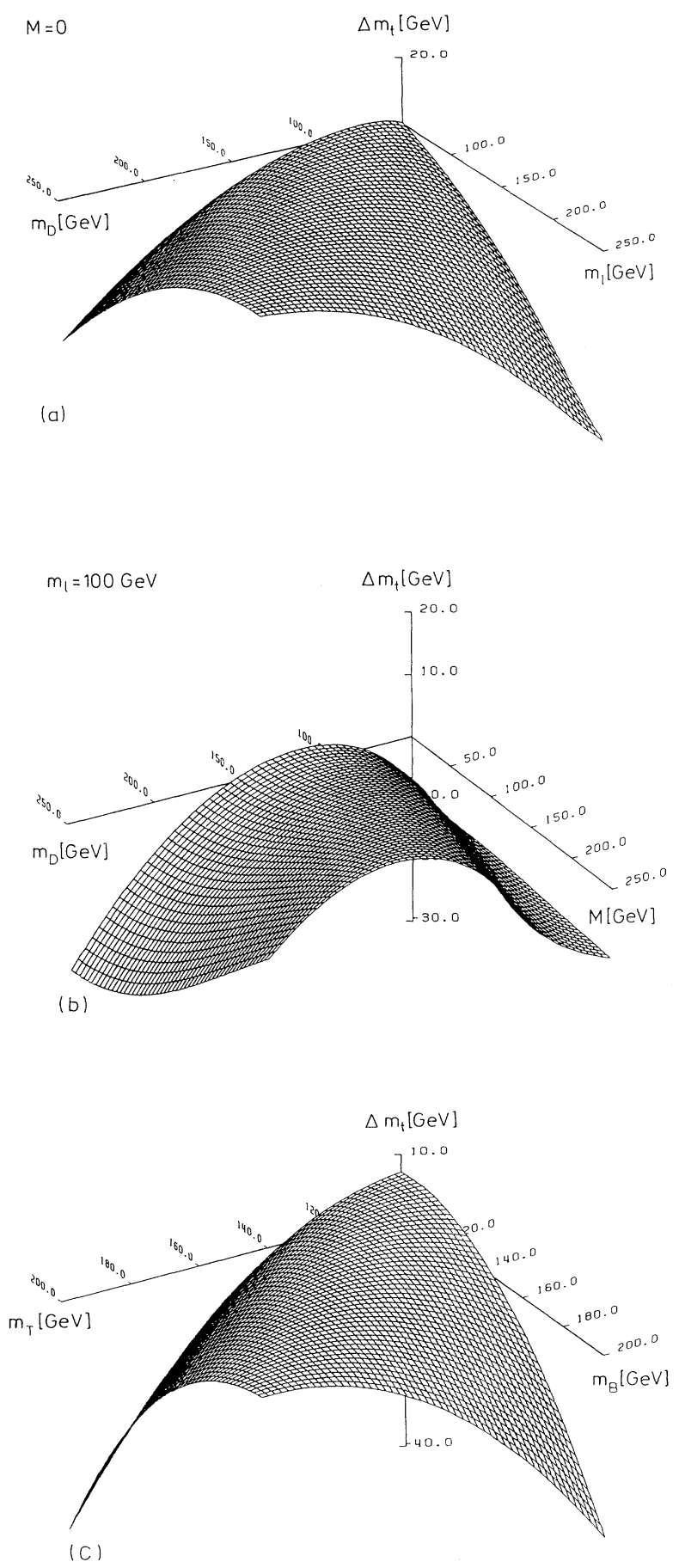

FIG. 6. Shift in the $m_{t}$ value compatible with the measured muon lifetime induced by novel leptons, $N_{1}, N_{2}$, and $l$, (a) as a function of $m_{D}$ and $m_{l}$, assuming $m_{1}=m_{2}=m_{D}$ (Dirac neutrino) and (b) as a function of $m_{D}=\sqrt{m_{1} m_{2}}$ and $M=m_{2}-m_{1}$, assuming $m_{l}=100 \mathrm{GeV}$ (Majorana neutrinos). (c) $m_{t}$ shift induced by novel quarks, $T$ and $B$, as a function of $m_{T}$ and $m_{B}$. The total $m_{T}$ shift can be estimated by adding the individual effects due to the leptons and quarks alone. The experimental result $M_{W}=80.14 \mathrm{GeV}[28]$ and the central value $M_{H}=250 \mathrm{GeV}$ have been assumed.

$$
S=\frac{N_{c}}{6 \pi}\left(1-Y \ln \frac{m_{T}^{2}}{m_{B}^{2}}\right),
$$

with $Y=\frac{1}{3}$ being the weak hypercharge of the $(T, B)$ doublet, one can glean that the quarkonic contribution to the $S$ parameter is now less positive. It is still true that values of $M$ below $1 \mathrm{TeV}$ are preferred experimentally.

Finally, we examine the shift induced by $N_{1}, N_{2}, l, T$, and $B$ in the $m_{t}$ value predicted from $M_{W}$ measurements [28] via the familiar analysis of $\Delta r$ [14] (see Fig. 6). A comprehensive discussion of $\Delta r$ within the standard model may be found in Ref. [29], from where the contribution due to a generic quark doublet may be extracted. The additional leptonic contribution to $\Delta r$ is easily obtained from Eqs. (2.1), (3.5), and (3.7). In the following, we shall take advantage of the fact that the leptonic and quarkonic sector contribute almost independently to the $m_{t}$ shift as long as the latter is much smaller than the absolute value of $m_{t}$. We may thus study these contributions separately. The lepton-induced $m_{t}$ shift is shown for $M=0$ as a function of $\left(m_{D}, m_{l}\right)$ in Fig. 5(a) and for $m_{l}=100$ $\mathrm{GeV}$ as a function of $\left(m_{D}, M\right)$ in Fig. 5(b). The quarkinduced $m_{t}$ shift is displayed in Fig. 5(c) as a function of $\left(m_{T}, m_{B}\right)$. We have assumed the central value $M_{H}=250$ $\mathrm{GeV}$; however, Fig. 5 is insensitive to the precise value of $M_{H}$. We see that the $m_{t}$ shift may be both positive and negative. While it is strongly bounded from above, it may take large negative values. Figures 5(a) and 5(c) may be understood qualitatively by observing that the $T$ parameter plays a dominant role in this type of analysis [cf. Eq. (2.5)] and that the isospin breaking in the new fermion doublets comes in at the expense of the mass splitting in the $(t, b)$ doublet. Similar observations have been made in Ref. [15].

\section{CONCLUSIONS}

We have calculated the contributions to $S, T$, and $U$ induced by a lepton family with Majorana neutrinos. A hypothetical heavy Majorana neutrino in the third generation decouples and cannot be constrained by comparing observable radiative corrections with experiment. However, such an analysis is possible for a new generation. Assuming that the standard model is extended by a fourth generation with Hill-Paschos-type leptons and conventional quarks, we have scanned the space of the new mass parameters and determined the ranges favored by a global analysis of the latest low-energy, LEP, and $M_{W}$ data. Provided that the isospin breaking in the quark sector is moderate, there is a considerable support of allowed Dirac and charged-lepton masses for Majorana masses $M$ way up to $1 \mathrm{TeV}$. The unfavorable effect of a more substantial mass splittings in the quark doublet can partly be compensated by choosing $M$ sufficiently large. However, if $M$ exceeds $1 \mathrm{TeV}$ by too much then the nonobservation of the lighter Majorana neutrino at LEP implies that the Dirac mass is pushed beyond its 
triviality bound. Moreover, fine-tuning problems enter the stage. Another interesting feature of the considered fourth-generation model is that the value of $m_{t}$ extracted from $M_{W}$ measurements can be substantially reduced as compared to the prediction within the minimal standard model.

\section{ACKNOWLEDGMENTS}

We are grateful to Wilfried Buchmüller and Apostolos Pilaftsis for instructive comments on Majorana neutrino phenomenology and to Alberto Sirlin for very useful communications regarding the formulation of observable radiative corrections.
[1] The LEP Collaborations: ALEPH, DELPHI, L3, and OPAL, Phys. Lett. B 276, 247 (1992).

[2] C. T. Hill and E. A. Paschos, Phys. Lett. B 241, 96 (1990).

[3] Particle Data Group, K. Hikasa et al., Phys. Rev. D 45, S1 (1992).

[4] W. Buchmüller, C. Greub, and H. -G. Kohrs, Nucl. Phys. B370, 3 (1992).

[5] W. Buchmüller and C. Greub, Phys. Lett. B 256, 465 (1991); Nucl. Phys. B363, 345 (1991).

[6] E. Ma and P. Roy, Phys. Rev. Lett. 68, 2879 (1992).

[7] B. W. Lynn, M. E. Peskin, and R. G. Stuart, SLAC Report No. SLAC-PUB-3725 (unpublished); also in Physics at LEP, LEP Jamboree, Geneva, Switzerland, 1985, edited by J. Ellis and R. Peccei (CERN Yellow Report No. 8602, Geneva, 1986), Vol. 1, p. 90.

[8] G. Burgers, F. Jegerlehner, B. Kniehl, and J. Kühn, in $Z$ Physics at LEP 1, Proceedings of the Workshop, Geneva, Switzerland, 1989, edited by G. Altarelli, R. Kleiss, and C. Verzegnassi (CERN Yellow Report No. 89-08, Geneva, 1989), Vol. 1, p. 55.

[9] D. C. Kennedy and B. W. Lynn, Nucl. Phys. B322, 1 (1989).

[10] M. E. Peskin and T. Takeuchi, Phys. Rev. Lett. 65, 964 (1990); Phys. Rev. D 46, 381 (1992).

[11] W. J. Marciano and J. L. Rosner, Phys. Rev. Lett. 65, 2963 (1990).

[12] D. C. Kennedy and P. Langacker, Phys. Rev. Lett. 65, 2967 (1990).

[13] G. Altarelli and R. Barbieri, Phys. Lett. B 253, 161 (1991).

[14] A. Sirlin, Phys. Rev. D 22, 971 (1980).

[15] S. Bertolini and A. Sirlin, Nucl. Phys. B248, 589 (1984);
Phys. Lett. B 257, 179 (1991).

[16] B. A. Kniehl, Nucl. Phys. B352, 1 (1991).

[17] J. Ellis, G. L. Fogli, and E. Lisi, Phys. Lett. B 285, 238 (1992); 292, 427 (1992).

[18] W. F. L. Hollik, Fortschr. Phys. 38, 165 (1990).

[19] B. A. Kniehl and A. Sirlin, Phys. Rev. D 47, 883 (1993).

[20] F. Jegerlehner, PSI Report No. PSI-PR-91-16, 1991 (unpublished); in Progress in Particle and Nuclear Physics, edited by A. Faessler (Pergamon Press, Oxford, 1991), Vol. 27, p. 1 .

[21] G. Bhattacharyya, S. Banerjee, and P. Roy, Phys. Rev. D 45, R729 (1992).

[22] A. Djouadi and C. Verzegnassi, Phys. Lett. B 195, 265 (1987); A. Djouadi, Nuovo Cimento 100A, 357 (1988); B. A. Kniehl, Nucl. Phys. B347, 86 (1990).

[23] D. A. Ross and M. Veltman, Nucl. Phys. B95, 135 (1975); M. Veltman, ibid. B123, 89 (1977).

[24] T. Appelquist and J. Carazzone, Phys. Rev. D 11, 2856 (1975).

[25] W. Grimus and H. Neufeld, Nucl. Phys. B325, 18 (1989).

[26] E. Gates and J. Terning, Phys. Rev. Lett. 67, 1840 (1991).

[27] N. Cabibbo, L. Maiani, G. Parisi, and R. Petronzio, Nucl. Phys. B158, 295 (1979).

[28] CDF Collaboration, F. Abe et al., Phys Rev. Lett. 65, 2243 (1990); Phys. Rev. D 43, 2070 (1991); UA2 Collaboration, J. Alitti et al., Phys. Lett. B 276, 354 (1992).

[29] G. Degrassi, S. Fanchiotti, and A. Sirlin, Nucl. Phys. B351, 49 (1991); F. Halzen and B. A. Kniehl, ibid. B353, 567 (1991); S. Fanchiotti, B. Kniehl, and A. Sirlin, this issue, Phys. Rev. D 48, 307 (1993). 